\title{
Battery Charger Device
}

National Cancer Institute

\section{Source}

National Cancer Institute. Battery Charger Device. NCI Thesaurus. Code C50346.

A device designed to restore the capacity of a battery. 